\title{
Effect of Polymerization Kinetics in the Pore Distribution in Polyacrylamide
}

\author{
Esmeralda Rodriguez-Miranda ${ }^{1}$, Edgar Omar Castrejon-Gonzalez ${ }^{2}$ and Gabriel Herrera-Perez ${ }^{3}$
}

1. Departamento de Medicina y Nutricion, Universidad de Guanajuato, Mexico

2. Departamento de Ingenieria Quimica, Instituto Tecnologico de Celaya, Mexico

3. Departamento de Ingenieria en Materiales, Instituto Tecnologico Superior de Irapuato, Mexico

Acrylamide or 2-propenamide is an amide-type organic compound from which is synthesized polyacrylamide polymer with multiple uses, in the cosmetic, paper, adhesives, in plastics production. Another application is given in experimental research in the area of biology and molecular sieve in the electrophoresis of proteins or nucleic acids. For this application, the polymer formation is performed by copolymerization of acrylamide and bisacrylamide (N, N'-methylene-bis-acrylamide), a reaction initiated by tetramethylethylenediamine (TEMED) and ammonium persulfate. Despite the widespread use of polyacrylamide gels / bis-acrylamide as a molecular sieve, it is important to understand the mechanism of polymerization to identify possible causes for not a gel is obtained with homogeneous pore distribution, elasticity and transparency.

Acrylamide is the organic amide compound is soluble in water, ethanol, methanol, dimethyl ether and acetone is not soluble in heptane and benzene. Its structure is composed of two linked acrylamide molecules by their amino groups (NH2), facing nonreactive polymerization. For this reason is the name of a, while if the vinyl group bonded $(\mathrm{C}=\mathrm{C})$ are called di-acrylamide. The polymerized bisacrylamide together with acrylamide and establishes bridges between the linear chains of polyacrylamide, and thus prevents sliding of the latter and leads to gel formatio.

In this paper we evaluate the kinetics of the polymerization reaction of acrylamide by Infrared Spectroscopy (FTIR). And they performed the characterization of pore structure and distribution of the polymers or gels using Scanning Electron Microscopy (SEM). Finally the gels were assessed molecular sieve synthesized as in the separation of membrane protein extracts of mammalian sperm plasma (pig).

The polymerization reaction is initiated by the formation of free radicals in the medium, which is achieved by addition to the medium of the generally called catalysts, although it would be more correct to call them initiators of the process. The following are commonly used: ammonium persulfate or riboflavin or riboflavin-5-phosphate in the presence of ultraviolet light of long wavelength (serving emitted by a fluorescent lamp) and traces of oxygen free radicals arises. In both cases an amine is further added to favor the formation of free radicals and is generally used $\mathrm{N}, \mathrm{N}, \mathrm{N}$ ', N'tetramethylethylenediamine (TEMED). On reacting acrylamide polymerization for electrophoresis, using persulphate ammonium and tetramethylethylenediamine (TEMED).

The spectra of the samples were performed in solution in an ATR (Attenuated Total Reflection), a special device of the spectrophotometer is used to obtain spectra of liquid samples. To obtain the spectrum of the background, was placed in $1 \mathrm{ml}$ of distilled water ATR with the aid of a pipette and the samples over a slide was placed. We used the same volume of acrylamide, bisacrylamide, TEMED, SDS, and ammonium persulfate tris the different $\mathrm{pH}$. Each spectrum of these solutions, the spectrophotometer automatically subtracts the background. 
In the analysis of the gels in the Scanning Electron Microscope (SEM), to a first approximation, the dried gels were used and wet $9 \%$ acrylamide / bisacrylamide $\mathrm{pH} 8.8$ in order to choose the best condition where the pores could be observed sharper. Figure 1 shows micrographs previously dry gel at different magnifications (central), seen in the image of the gel surface morphology at 1000X, in the upper left image of the cavities increase observed in 10000X and not uncertain question of pores and is important to note that increases 40000X pores are observed between 50 and $100 \mathrm{~nm}$ right superior sharpness however is very low, possibly due to damage suffered by the sample.

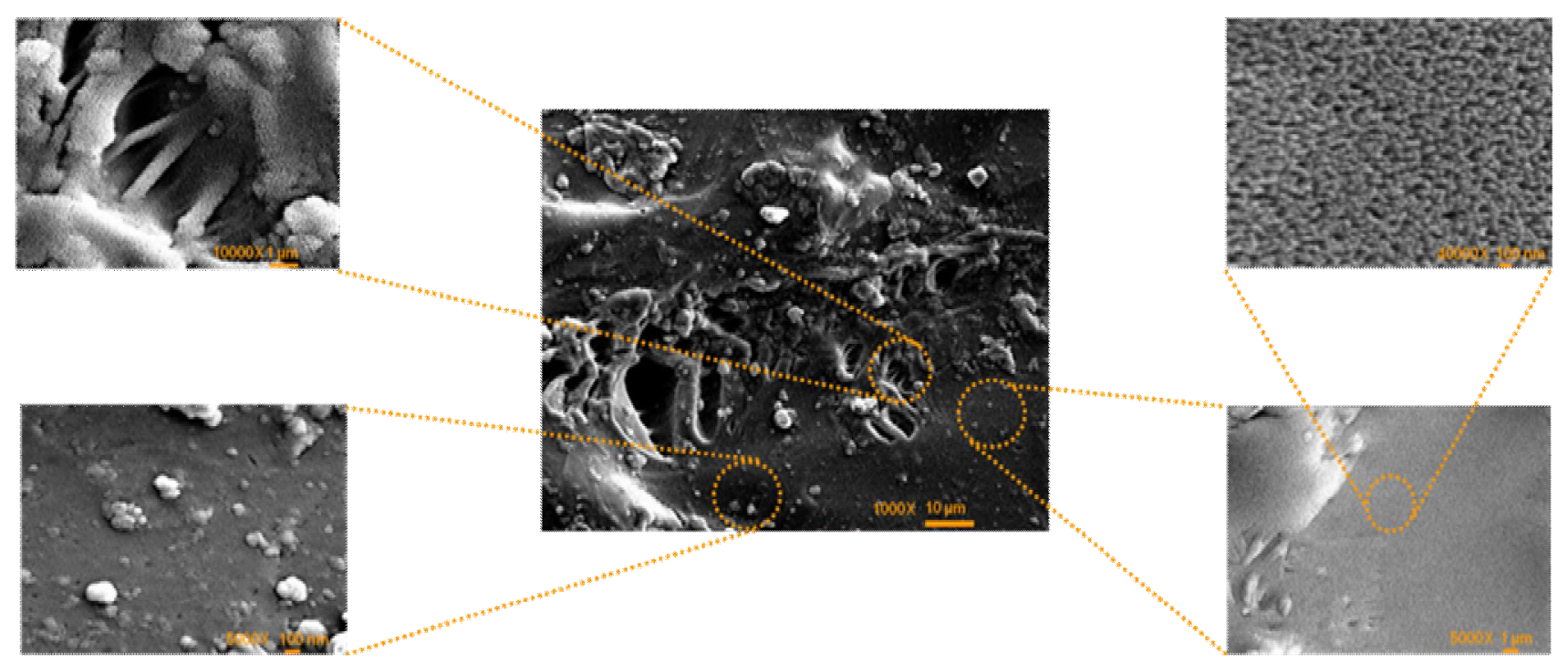

Figure 2. Shows micrographs wet gel at different magnifications showing the effect of spent drying process and pretreatment of the sample during analysis. 